\title{
Numerical Solutions to Optimal Portfolio Selection and Consumption Strategies under Stochastic Volatility
}

\author{
Lei Ge $\mathbb{D}^{1}$ and Qiang Zhang ${ }^{2}$ \\ ${ }^{1}$ School of Finance, Southwestern University of Finance and Economics, Chengdu, China \\ ${ }^{2}$ Department of Mathematics, City University of Hong Kong, Hong Kong \\ Correspondence should be addressed to Lei Ge; leige365@outlook.com
}

Received 23 April 2020; Revised 30 June 2020; Accepted 13 July 2020; Published 30 July 2020

Guest Editor: Lei Xie

Copyright ( 2020 Lei Ge and Qiang Zhang. This is an open access article distributed under the Creative Commons Attribution License, which permits unrestricted use, distribution, and reproduction in any medium, provided the original work is properly cited.

\begin{abstract}
Based on the method of dynamic programming, this paper uses analysis methods governed by the nonlinear and inhomogeneous partial differential equation to study modern portfolio management problems with stochastic volatility, incomplete markets, limited investment scope, and constant relative risk aversion (CRRA). In this paper, a three-level Crank-Nicolson finite difference scheme is used to determine numerical solutions under this general setting. One of the main contributions of this paper is to apply this three-level technology to solve the portfolio selection problem. In addition, we have used a technique to deal with the nonlinear term, which is another novelty in performing the Crank-Nicolson algorithm. The Crank-Nicolson algorithm has also been extended to third-order accuracy by performing Richardson's extrapolation. The accuracy of the proposed algorithm is much higher than the traditional finite difference method. Lastly, experiments are conducted to show the performance of the proposed algorithm.
\end{abstract}

\section{Introduction}

How to optimally allocate assets and optimally consume are extremely important and difficult topics in portfolio management [1-3]. These topics are important not only for theoretical consideration but also for applications in the financial industry. Early studies usually assumed the volatility of the risky asset to be a constant. However, in recent years, researchers found that volatility should be modeled as stochastic rather than deterministic [4-7]. This adds further complication to the problem. The optimal asset allocation and optimal consumption strategies are governed by the Hamilton-Jacobi-Bellman (HJB) equation. Due to the nonlinearity and inhomogeneity of this partial differential equation, no exact solution has been found. Furthermore, even numerical solutions are not available. In this paper, we present an accurate and efficient numerical method for solving this equation and generate the first set of accurate numerical solutions for this problem.
Due to the importance of portfolio selection under stochastic volatilities, several important theoretical works have been carried out, and exact solutions have been obtained under certain special settings, such as no consumption [8-10], complete markets which means that the stock movement and the volatility movement are either perfectly correlated or perfectly anticorrelated [9-12], or when investors have unit elasticity of intertemporal substitution of consumption [13].

In this paper, we consider this optimal stochastic control problem under a general setting: stochastic volatility, incomplete markets, finite investment horizons, and CRRA utility. Our numerical method combines a threelevel Crank-Nicolson scheme and Richardson's extrapolation technique. The Crank-Nicolson scheme has secondorder accuracy in terms of discretization error, and Richardson's extrapolation technique further improves the accuracy. We verify that our numerical method is accurate and efficient. 
This paper is organized as follows. In Section 2, we describe the model for financial market, the stochastic control optimization procedure, and the governing HJB equation for the optimal asset allocation and consumption strategies. In Section 3, we present our numerical method for solving the HJB equation. In Section 4, we verify the accuracy and the efficiency of our numerical method and present accurate numerical solutions for the optimal asset allocation strategy and the optimal consumption strategy. In the last section, we present our conclusions.

\section{Financial Market and Stochastic Control}

We consider a market consisting of one riskless asset $B_{t}$, whose price is governed by

$$
\mathrm{d} B_{t}=r B_{t} \mathrm{~d} t
$$

with a constant risk-free interest rate $r$ and a risky asset $S_{t}$ modeled as

$$
\mathrm{d} S_{t}=S_{t}\left[\mu\left(v_{t}\right)+\sigma\left(v_{t}\right) \mathrm{d} W_{t}^{S}\right]
$$

In (2), $\mu\left(v_{t}\right)$ and $\sigma\left(v_{t}\right)$ are the return and the stochastic volatility of the stock price $S_{t}$, respectively. $v_{t}=\sigma_{t}^{2}$ is the stochastic variance of $S_{t}$. Empirical studies show presence of mean reversion in the stock movements [14]. Heston model [5] is selected for $v_{t}$, namely,

$$
\mathrm{d} v_{t}=\kappa\left(\theta-v_{t}\right) \mathrm{d} t+\xi \sqrt{v_{t}} \mathrm{~d} W_{t}^{v} .
$$

Here, $\mathrm{d} W_{t}^{v}$ and $\mathrm{d} W_{t}^{S}$ are the increments of the Wiener processes under a probability $P$. The correlation between $\mathrm{d} W_{t}^{v}$ and $\mathrm{d} W_{t}^{S}$ is $\rho$, namely, Corr $\left(\left(\mathrm{d} v_{t} / v_{t}\right),\left(\mathrm{d} S_{t} / S_{t}\right)\right)=\rho \mathrm{d} t$. We assume $\rho$ is a constant. In (3), $\theta$ is the long-run average variance (i.e., as $t$ tends to infinity, the expected value of $v_{t}$ tends to $\theta$ ), $\kappa$ is the rate at which $v_{t}$ reverts to $\theta$, and $\xi$ is the volatility of the stock variance $v_{t}$. The parameters $\kappa, \theta, \xi$ are positive constants and need to satisfy the Feller condition, $2 \kappa \theta>\xi^{2}$, to ensure that $v_{t}$ is strictly positive. The risk premia is defined as

$$
A=\frac{\mu_{t}-r}{\sigma_{t}^{2}} \equiv \frac{\mu_{t}-r}{v_{t}}
$$

Following $[1,5,15-17]$, we assume $A$ is a constant. This means the stock excess return is proportional to the stock variance.

Consider an investor who has an initial wealth $w_{0}$ and needs to determine strategies for asset allocation and consumption over an investment horizon $[0, T]$. Let $w_{t}$ be the investor's wealth at time $t$. The strategies consist of an asset allocation rate $\varphi_{t}$ and a consumption rate $c_{t}$, which mean he/she allocates $\varphi_{t} w_{t}$ to the risky asset and $\left(1-\varphi_{t}\right) w_{t}$ to the riskless asset at time $t$ and consumes $c_{t} \mathrm{~d} t$ over the time interval $[t, t+\mathrm{d} t]$. Thus, under the strategies $\varphi_{t}$ and $c_{t}$, the wealth process is governed by

$$
\mathrm{d} w_{t}=\frac{\varphi_{t} w_{t}}{S_{t}} \mathrm{~d} S_{t}+\left(1-\varphi_{t}\right) w_{t} r \mathrm{~d} t-c_{t} \mathrm{~d} t
$$

The goal is to maximize the expected utilities over the investment horizon, namely,

$$
\sup _{\varphi_{t}, c_{t}} E\left[\int_{0}^{T} \alpha e^{-\beta t} u_{1}\left(c_{t}\right) \mathrm{d} t+(1-\alpha) e^{-\beta T} u_{2}\left(w_{T}\right)\right]
$$

In (6), $\varphi_{t}$ and $c_{t}$ are control variables for this optimization problem. $E$ is the expectation operator under the probability $P$. $\beta$ is the subjective discount rate, namely, the time preference of the investor. The larger $\beta$ is, the more weight the investor puts on the present than on the future. The parameter $\alpha$ determines the relative importance between intertemporal consumption and the terminal wealth. $u_{1}(\cdot)$ and $u_{2}(\cdot)$ are the investor's utility functions which measure the investor's degree of satisfaction with the outcomes from intertemporal consumption and terminal wealth, respectively.

CRRA utility functions have been widely adopted for modeling investors' behavior. Therefore, we adopt the CRRA utility function for $u_{1}(\cdot)$ and $u_{2}(\cdot)$ :

$$
\begin{aligned}
& \begin{cases}u_{1}\left(c_{t}\right)=\frac{a_{c} c_{t}^{1-\gamma}}{1-\gamma}, & \text { for } \gamma \neq 1, \\
a_{c} \log \left(c_{t}\right), & \text { for } \gamma=1,\end{cases} \\
& \begin{cases}u_{2}\left(w_{T}\right)=\frac{a_{w} w_{T}^{1-\gamma}}{1-\gamma}, & \text { for } \gamma \neq 1, \\
a_{w} \log \left(w_{T}\right), & \text { for } \gamma=1,\end{cases}
\end{aligned}
$$

where $\gamma, a_{c}$, and $a_{w}$ are positive constants. Since $u_{1}(\cdot)$ and $u_{2}(\cdot)$ stand for the intertemporal consumption utility and the terminal wealth utility of the same investor, we use the same $\gamma$ in $u_{1}(\cdot)$ and $u_{2}(\cdot)$. However, $a_{c}$ and $a_{w}$ can be different since $c$ and $w$ have different dimensions.

Let $V(t, w, v)$ be the value function of problem (6), which is given by

$$
\begin{aligned}
V\left(t, w_{t}, v_{t}\right)= & \sup _{\varphi_{t}, c_{t}} E\left[\int_{t}^{T} \alpha e^{-\beta t^{\prime}} u_{1}\left(c_{t}^{\prime}\right) \mathrm{d} t^{\prime}\right. \\
& \left.+(1-\alpha) e^{-\beta T} u_{2}\left(w_{T}\right) \mid \mathscr{F}_{t}\right],
\end{aligned}
$$

where $\mathscr{F}_{T}$ is the filtration associated to the stochastic processes in this problem. The terminal condition is obtained by setting $t=T$ in (8):

$$
V\left(T, w_{T}, v_{T}\right)=(1-\alpha) e^{-\beta T} u_{2}\left(w_{T}\right) .
$$

Based on the HJB dynamic programming procedure, $V$ is governed by

$$
\begin{aligned}
0= & \sup _{\varphi, c}\left[\alpha e^{-\beta t} u_{1}(c)+V_{t}+(r w+\varphi w A v-c) V_{w}\right. \\
& \left.+\kappa(\theta-v) V_{v}+\frac{1}{2} \varphi^{2} w^{2} v V_{w w}+\varphi w \rho \xi v V_{w v}+\frac{1}{2} \xi^{2} v V_{v v}\right],
\end{aligned}
$$


with the optimal strategies $\varphi^{*}$ and $c^{*}$ determined by

$$
\begin{gathered}
\varphi^{*}=-\frac{A V_{w}+\rho \xi V_{w v}}{w V_{w w}}, \\
c^{*}=\left(\frac{V_{w}}{a_{c} \alpha e^{-\beta t}}\right)^{-(1 / \gamma)} .
\end{gathered}
$$

After substituting expressions (11) and (12) into (10), one obtains an equation for the value function $V$ :

$$
\begin{aligned}
& \frac{\gamma}{1-\gamma}\left(a_{c} \alpha e^{-\beta t}\right)^{(1 / \gamma)} V_{w}^{(1-\gamma /-\gamma)}+r w V_{w}+V_{t}+\kappa(\theta-v) V_{v} \\
& +\frac{1}{2} \xi^{2} v V_{v v}-\frac{1}{2} v \frac{\left(A V_{w}+\rho \xi V_{w v}\right)^{2}}{V_{w w}}=0 .
\end{aligned}
$$

Based on the terminal condition and the scaling property of (13), it is reasonable to guess that

$$
V(\tau, w, v)=e^{-\beta(T-\tau)} \frac{a_{w} w^{1-\gamma}}{1-\gamma} f(\tau, v)^{\gamma},
$$

where $\tau=T-t$, (13) becomes

$$
\begin{aligned}
0= & -f_{\tau}+\frac{1}{2} \xi^{2} v f_{v v}+\left(\kappa(\theta-v)+\frac{1-\gamma}{\gamma} A \rho \xi v\right) f_{v} \\
& -\frac{1}{2}(1-\gamma)\left(1-\rho^{2}\right) \xi^{2} v \frac{f_{v}^{2}}{f} \\
& +\left(\frac{(1-\gamma) A^{2}}{2 \gamma^{2}} v+\frac{(1-\gamma) r}{\gamma}-\frac{\beta}{\gamma}\right) f+\left(\frac{\alpha a_{c}}{a_{w}}\right)^{(1 / \gamma)},
\end{aligned}
$$

with

$$
f(0, v)=(1-\alpha)^{(1 / \gamma)},
$$

and (11) and (12) become

$$
\begin{aligned}
& \varphi^{*}=\frac{A}{\gamma}+\rho \xi \frac{f_{v}}{f}, \\
& \frac{c^{*}}{w}=\left(\frac{\alpha a_{c}}{a_{w}}\right)^{(1 / \gamma)} f^{-1} .
\end{aligned}
$$

Equation (15) is a nonlinear and inhomogeneous partial differential equation. Since no closed-form solution is available for this equation, numerical computation plays a critical role for studying this important practical problem in modern finance. However, there are even no numerical solutions available in the literature.

\section{Numerical Method}

In this section, we develop a numerical method for solving (15). For the sake of conciseness of our expressions, we rewrite (15) as

$$
\begin{aligned}
& -f_{\tau}+a_{1} v f_{v v}+\left(a_{2} v+a_{3}\right) f_{v}+a_{4} v \frac{f_{v}^{2}}{f}+\left(a_{5} v+a_{6}\right) f \\
& +\alpha^{(1 / \gamma)} a_{7}=0
\end{aligned}
$$

with the initial condition $f(0, v)=(1-\alpha)^{(1 / \gamma)}$, where

$$
\begin{aligned}
& a_{1}=\frac{1}{2} \xi^{2}, \\
& a_{2}=\frac{1-\gamma}{\gamma} A \rho \xi-\kappa, \\
& a_{3}=\kappa \theta, \\
& a_{4}=-\frac{1}{2}(1-\gamma) \xi^{2}\left(1-\rho^{2}\right), \\
& a_{5}=\frac{1-\gamma}{2 \gamma^{2}} A^{2}, \\
& a_{6}=\frac{(1-\gamma) r}{\gamma}-\frac{\beta}{\gamma}, \\
& a_{7}=\left(\frac{a_{c}}{a_{w}}\right)^{(1 / \gamma)} .
\end{aligned}
$$

3.1. Crank-Nicolson Scheme and Richardson's Extrapolation. We use a three-level Crank-Nicolson scheme (see [18-21]) of second-order accuracy to solve the nonlinear and inhomogeneous partial differential equation given by (19) and use Richardson's extrapolation technique for further improving accuracy. Numerically, one can only solve (19) over a finite domain $v \in\left[0, v_{\max }\right]$. Since the boundary conditions at $v=0$ and at $v=v_{\max }$ are not known, we use one-sided difference method at these two numerical boundaries. Step sizes $\Delta \tau$ and $\Delta v$ are used to discretize $\tau$ and $v$, respectively. Thus, $\tau=n \Delta \tau$ and $v=m \Delta v$. We adopt the standard notation $f_{m}^{n}=f(n \Delta \tau, m \Delta v)$.

The three-level Crank-Nicolson scheme involves the levels $n-1, n$, and $n+1$. It is straightforward to discretize all linear terms in (19) with second-order errors, namely,

$$
\begin{aligned}
\left(f_{\tau}\right)_{m}^{n}= & \frac{f_{m}^{n+1}-f_{m}^{n-1}}{2 \Delta \tau}+O\left(\Delta \tau^{2}\right), \\
\left(f_{v}\right)_{m}^{n}= & \frac{1}{2}\left(\frac{f_{m+1}^{n+1}-f_{m-1}^{n+1}}{2 \Delta v}+\frac{f_{m+1}^{n-1}-f_{m-1}^{n-1}}{2 \Delta v}\right)+O\left(\Delta \tau^{2}\right) \\
& +O\left(\Delta v^{2}\right), \\
\left(f_{v v}\right)_{m}^{n}= & \frac{1}{2}\left(\frac{f_{m+1}^{n+1}-2 f_{m}^{n+1}+f_{m-1}^{n+1}}{\Delta v^{2}}+\frac{f_{m+1}^{n-1}-2 f_{m}^{n-1}+f_{m-1}^{n-1}}{\Delta v^{2}}\right) \\
& +O\left(\Delta \tau^{2}\right)+O\left(\Delta v^{2}\right) .
\end{aligned}
$$


The nonlinear term $f_{v}^{2} / f$ has two factors $f_{v} / f$ and $f_{v}$. We discretize the factor $f_{v} / f$ at level $n$ and approximate the factor $f_{v}$ as an average between $f_{v}$ at level $n-1$ and that at level $n+1$, namely,

$$
\begin{aligned}
\left(\frac{f_{v}^{2}}{f}\right)_{m}^{n}= & \left(\frac{f_{v}}{f}\right)_{m}^{n} \frac{1}{2}\left(\left(f_{v}\right)_{m}^{n+1}+\left(f_{v}\right)_{m}^{n-1}\right)+O\left(\Delta \tau^{2}\right) \\
= & \frac{f_{m+1}^{n}-f_{m-1}^{n}}{4 \Delta v f_{m}^{n}}\left(\frac{f_{m+1}^{n+1}-f_{m-1}^{n+1}}{2 \Delta v}+\frac{f_{m+1}^{n-1}-f_{m-1}^{n-1}}{2 \Delta v}\right) \\
& +O\left(\Delta \tau^{2}\right)+O\left(\Delta v^{2}\right) .
\end{aligned}
$$

This discretization scheme leads to a set of linear equations. Based on the expressions given by (21) and (22), equation (19) can be discretized as

$$
\begin{aligned}
e_{1}(m) f_{m-1}^{n+1}+e_{2}(m) f_{m}^{n+1}+e_{3}(m) f_{m+1}^{n+1}= & e_{4}(m)+O\left(\Delta \tau^{2}\right) \\
& +O\left(\Delta v^{2}\right),
\end{aligned}
$$

for $0<m<M$ and $n \geq 2$, where $M$ is the maximal value of $m$ and

$$
\begin{aligned}
e_{1}(m)= & \frac{a_{1} m}{\Delta v}-\frac{\lambda(m)}{2 \Delta v}, \\
e_{2}(m)= & \frac{-2 a_{1} m}{\Delta v}-\frac{1}{\Delta \tau}+a_{5} m \Delta v+a_{6}, \\
e_{3}(m)= & \frac{a_{1} m}{\Delta v}+\frac{\lambda(m)}{2 \Delta v}, \\
e_{4}(m)= & -\frac{1}{\Delta \tau} f_{m}^{n-1}-2 \alpha^{(1 / \gamma)} a_{7}-\frac{a_{1} m}{\Delta v}\left(f_{m+1}^{n-1}-2 f_{m}^{n-1}+f_{m-1}^{n-1}\right) \\
& -\frac{\lambda(m)}{2 \Delta v}\left(f_{m+1}^{n-1}-f_{m-1}^{n-1}\right)-\left(a_{5} m \Delta v+a_{6}\right) f_{m}^{n-1},
\end{aligned}
$$

with

$$
\lambda(m)=a_{2} m \Delta v+a_{3}+\frac{a_{4} m\left(f_{m+1}^{n}-f_{m-1}^{n}\right)}{2 f_{m}^{n}} .
$$

Since (23) is not applicable to the boundaries at $m=0$ and $m=M$, we used one-sided difference technique to discretize (19) and obtained the boundary equations in the following. It is straightforward to show that, at $m=0$, we have

$$
\begin{aligned}
& f_{v}(0, \tau)=\frac{1}{4 \Delta v}\left(-3 f_{0}^{n+1}+4 f_{1}^{n+1}-f_{2}^{n+1}-3 f_{0}^{n-1}+4 f_{1}^{n-1}-f_{2}^{n-1}\right)+O\left(\Delta \tau^{2}\right)+O\left(\Delta v^{2}\right), \\
& f_{v v}(0, \tau)=\frac{1}{2 \Delta v^{2}}\left(2 f_{0}^{n+1}-5 f_{1}^{n+1}+4 f_{2}^{n+1}-f_{3}^{n+1}+2 f_{0}^{n-1}-5 f_{1}^{n-1}+4 f_{2}^{n-1}-f_{3}^{n-1}\right)+O\left(\Delta \tau^{2}\right)+O\left(\Delta v^{2}\right), \\
& \frac{f_{v}^{2}}{f}(0, \tau)=\frac{-3 f_{0}^{n}+4 f_{1}^{n}-f_{2}^{n}}{8 \Delta v^{2} f_{0}^{n}}\left(-3 f_{0}^{n+1}+4 f_{1}^{n+1}-f_{2}^{n+1}-3 f_{0}^{n-1}+4 f_{1}^{n-1}-f_{2}^{n-1}\right)+O\left(\Delta \tau^{2}\right)+O\left(\Delta v^{2}\right),
\end{aligned}
$$

and at $m=M$, we have

$$
\begin{aligned}
& f_{v}\left(v_{\max }, \tau\right)=\frac{1}{4 \Delta v}\left(3 f_{M}^{n+1}-4 f_{M-1}^{n+1}+f_{M-2}^{n+1}+3 f_{M}^{n-1}-4 f_{M-1}^{n-1}+f_{M-2}^{n-1}\right)+O\left(\Delta \tau^{2}\right)+O\left(\Delta v^{2}\right), \\
& f_{v v}\left(v_{\max }, \tau\right)=\frac{1}{2 \Delta v^{2}}\left(2 f_{M}^{n+1}-5 f_{M-1}^{n+1}+4 f_{M-2}^{n+1}-f_{M-3}^{n+1}+2 f_{M}^{n-1}-5 f_{M-1}^{n-1}+4 f_{M-2}^{n-1}-f_{M-3}^{n-1}\right)+O\left(\Delta \tau^{2}\right)+O\left(\Delta v^{2}\right), \\
& \frac{f_{v}^{2}}{f}\left(v_{\max }, \tau\right)=\frac{3 f_{M}^{n}-4 f_{M-1}^{n}+f_{M-2}^{n}}{8 \Delta v^{2} f_{M}^{n}}\left(3 f_{M}^{n+1}-4 f_{M-1}^{n+1}+f_{M-2}^{n+1}+3 f_{M}^{n-1}-4 f_{M-1}^{n-1}+f_{M-2}^{n-1}\right)+O\left(\Delta \tau^{2}\right)+O\left(\Delta v^{2}\right) .
\end{aligned}
$$

By substituting these expressions into (19), we have

$$
\begin{array}{r}
d_{1}(0) f_{0}^{n+1}+d_{2}(0) f_{1}^{n+1}+d_{3}(0) f_{2}^{n+1}+d_{4}(0) f_{3}^{n+1}=d_{5}(0)+O\left(\Delta \tau^{2}\right)+O\left(\Delta v^{2}\right), \\
d_{4}(M) f_{M-3}^{n+1}+d_{3}(M) f_{M-2}^{n+1}+d_{2}(M) f_{M-1}^{n+1}+d_{1}(M) f_{M}^{n+1}=d_{5}(M)+O\left(\Delta \tau^{2}\right)+O\left(\Delta v^{2}\right),
\end{array}
$$


where

$$
\begin{aligned}
d_{1}(0)= & -\frac{3 \lambda(0)}{2 \Delta v}-\frac{1}{\Delta \tau}+a_{6}, \\
d_{2}(0)= & \frac{2 \lambda(0)}{\Delta v}, \\
d_{3}(0)= & -\frac{\lambda(0)}{2 \Delta v}, \\
d_{4}(0)= & 0 \\
d_{5}(0)= & -\frac{1}{\Delta \tau} f_{0}^{n-1}-2 \alpha^{\frac{1}{\gamma}} a_{7}-\frac{\lambda(0)}{2 \Delta v}\left(-3 f_{0}^{n-1}+4 f_{1}^{n-1}-f_{2}^{n-1}\right)-a_{6} f_{0}^{n-1}, \\
d_{1}(M)= & \frac{2 a_{1} M}{\Delta v}+\frac{3 \lambda(M)}{2 \Delta v}-\frac{1}{\Delta \tau}+a_{5} M \Delta v+a_{6}, \\
d_{2}(M)= & \frac{-5 a_{1} M}{\Delta v}-\frac{2 \lambda(M)}{\Delta v}, \\
d_{3}(M)= & \frac{4 a_{1} M}{\Delta v}+\frac{\lambda(M)}{2 \Delta v}, \\
d_{4}(M)= & \frac{-a_{1} M}{\Delta v}, \\
d_{5}(M)= & -\frac{1}{\Delta \tau} f_{M}^{n-1}-2 \alpha^{(1 / \gamma)} a_{7}-\frac{a_{1} M}{\Delta v}\left(2 f_{M}^{n-1}-5 f_{M-1}^{n-1}+4 f_{M-2}^{n-1}-f_{M-3}^{n-1}\right) \\
& -\frac{\lambda(M)}{2 \Delta v}\left(3 f_{M}^{n-1}-4 f_{M-1}^{n-1}+f_{M-2}^{n-1}\right)-\left(a_{5} M \Delta v+a_{6}\right) f_{M}^{n-1},
\end{aligned}
$$

with

$$
\begin{aligned}
\lambda(0) & =a_{3}, \\
\lambda(M) & =a_{2} M \Delta v+a_{3}+\frac{a_{4} M\left(3 f_{M}^{n}-4 f_{M-1}^{n}+f_{M-2}^{n}\right)}{2 f_{M}^{n}} .
\end{aligned}
$$

From (23), (28), and (29), the numerical solution of (19) for $n \geq 2$ is determined by the following system of linear equations:

$$
\left[\begin{array}{cccccc}
d_{1}(0) & d_{2}(0) & d_{3}(0) & d_{4}(0) & & \\
e_{1}(1) & e_{2}(1) & e_{3}(1) & & & \\
& e_{1}(2) & e_{2}(2) & e_{3}(2) & & \\
& & \ddots & \ddots & \ddots & \\
& & & e_{1}(M-1) & e_{2}(M-1) & e_{3}(M-1) \\
& & d_{4}(M) & d_{3}(M) & d_{2}(M) & d_{1}(M)
\end{array}\right]\left[\begin{array}{c}
f_{0}^{n+1} \\
f_{1}^{n+1} \\
f_{2}^{n+1} \\
\vdots \\
f_{M-1}^{n+1} \\
f_{M}^{n+1}
\end{array}\right]=\left[\begin{array}{c}
d_{5}(0) \\
e_{4}(1) \\
e_{4}(2) \\
\vdots \\
e_{4}(M-1) \\
d_{5}(M)
\end{array}\right] .
$$


After eliminating $d_{3}(0), d_{4}(0), d_{3}(M)$, and $d_{4}(M),(33)$ can be transformed into the following tridiagonal matrix form for $n \geq 2$ :

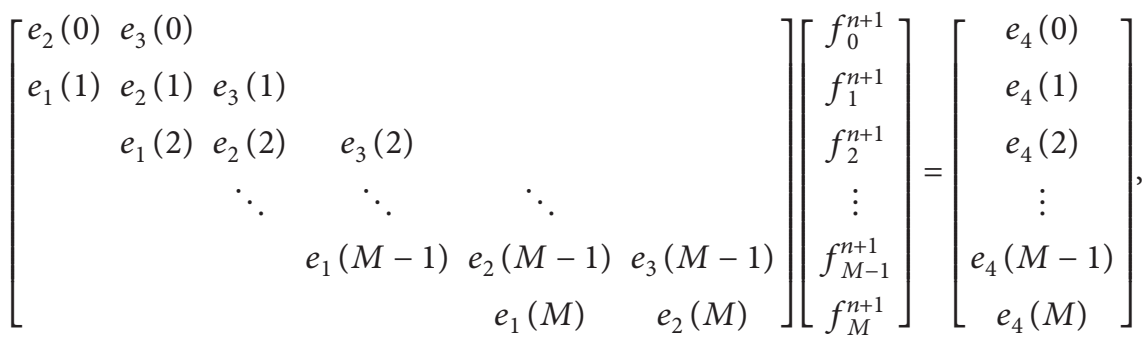

where

$$
\begin{aligned}
& e_{2}(0)=d_{1}(0)-\frac{e_{3}(2) d_{3}(0)-e_{2}(2) d_{4}(0)}{e_{3}(2) e_{3}(1)} e_{1}(1), \\
& e_{3}(0)=d_{2}(0)-\frac{d_{4}(0)}{e_{3}(2)} e_{1}(2)-\frac{e_{3}(2) d_{3}(0)-e_{2}(2) d_{4}(0)}{e_{3}(2) e_{3}(1)} e_{2}(1), \\
& e_{4}(0)=d_{5}(0)-\frac{d_{4}(0)}{e_{3}(2)} e_{4}(2)-\frac{e_{3}(2) d_{3}(0)-e_{2}(2) d_{4}(0)}{e_{3}(2) e_{3}(1)} e_{4}(1), \\
& e_{1}(M)=d_{2}(M)-\frac{d_{4}(M)}{e_{1}(M-2)} e_{3}(M-2)-\frac{e_{1}(M-2) d_{3}(M)-e_{2}(M-2) d_{4}(M)}{e_{1}(M-2) e_{1}(M-1)} e_{2}(M-1), \\
& e_{2}(M)=d_{1}(M)-\frac{e_{1}(M-2) d_{3}(M)-e_{2}(M-2) d_{4}(M)}{e_{1}(M-2) e_{1}(M-1)} e_{3}(M-1), \\
& e_{4}(M)=d_{5}(M)-\frac{d_{4}(M)}{e_{1}(M-2)} e_{4}(M-2)-\frac{e_{1}(M-2) d_{3}(M)-e_{2}(M-2) d_{4}(M)}{e_{1}(M-2) e_{1}(M-1)} e_{4}(M-1) .
\end{aligned}
$$

The method given by (21) and (22) is a two-step method, namely, $f^{n+1}$ depends on $f^{n}$ and $f^{n-1}$. At the zeroth step, $f^{0}$ is given by initial condition (16), namely, $f_{m}^{0}=(1-\alpha)^{(1 / \gamma)}$, for $0 \leq m \leq M$. We now determine $f^{1}$, the solution at the first step. Performing Taylor expansion on $f(\Delta \tau, v)$ at $\tau=0$ gives

$$
\begin{aligned}
f(\Delta \tau, v)= & f(0, v)+f_{\tau}(0, v) \Delta \tau+\frac{1}{2} f_{\tau \tau}(0, v) \Delta \tau^{2} \\
& +\frac{1}{6} f_{\tau \tau \tau}(0, v) \Delta \tau^{3}+O\left(\Delta \tau^{4}\right),
\end{aligned}
$$

$$
\begin{aligned}
f_{\tau}(0, v)= & \left(a_{5} v+a_{6}\right) f(0, v)+\alpha^{(1 / \gamma)} a_{7} \\
f_{\tau \tau}(0, v)= & \left(a_{5}^{2} v^{2}+\left(a_{2} a_{5}+2 a_{5} a_{6}\right) v+a_{3} a_{5}+a_{6}^{2}\right) f(0, v)+\left(a_{5} v+a_{6}\right) \alpha^{(1 / \gamma)} a_{7}, \\
f_{\tau \tau \tau}(0, v)= & {\left[a_{5}^{3} v^{3}+\left(3 a_{2} a_{5}^{2}+3 a_{6} a_{5}^{2}\right) v^{2}+\left(a_{5} a_{2}^{2}+3 a_{5} a_{6} a_{2}+2 a_{1} a_{5}^{2}+3 a_{3} a_{5}^{2}+2 a_{4} a_{5}^{2}+3 a_{5} a_{6}^{2}\right) v+a_{2} a_{3} a_{5}+3 a_{3} a_{6} a_{5}+a_{6}^{3}\right] } \\
& \cdot f(0, v)+\left(a_{2} v+a_{3}\right) a_{5} \alpha^{(1 / \gamma)} a_{7}+\left(a_{5} v+a_{6}\right)^{2} \alpha^{(1 / \gamma)} a_{7} .
\end{aligned}
$$


The details of derivations for $f_{\tau}(0, v), f_{\tau \tau}(0, v)$, and $f_{\tau \tau \tau}(0, v)$ are given in Appendix A. From (36), $f_{m}^{1}$ is given by

$$
\begin{aligned}
f_{m}^{1}= & f_{m}^{0}+f_{\tau}(0, m \Delta v) \Delta \tau+\frac{1}{2} f_{\tau \tau}(0, m \Delta v) \Delta \tau^{2} \\
& +\frac{1}{6} f_{\tau \tau \tau}(0, m \Delta v) \Delta \tau^{3},
\end{aligned}
$$

with an error of $O\left(\Delta \tau^{4}\right)$.

Knowing $f$, the numerical solutions of optimal portfolio and consumption rules can be obtained from (17) and (18):

$$
\begin{aligned}
\varphi_{m}^{n *} & =\frac{A}{\gamma}+\rho \xi \frac{\left(f_{v}\right)_{m}^{n}}{f_{m}^{n}}, \\
\frac{c_{m}^{n *}}{w} & =\left(\frac{\alpha a_{c}}{a_{w}}\right)^{(1 / \gamma)}\left(f_{m}^{n}\right)^{-1},
\end{aligned}
$$

for $0 \leq m \leq M$, where $f_{m}^{n}$ is given by (34) and $\left(f_{v}\right)_{m}^{n}$ is given by

$$
\left(f_{v}\right)_{m}^{n}= \begin{cases}\frac{-3 f_{0}^{n}+4 f_{1}^{n}-f_{2}^{n}}{2 \Delta v}, & \text { for } m=0 \\ \frac{f_{m+1}^{n}-f_{m-1}^{n}}{2 \Delta v}, & \text { for } 0<m<M \\ \frac{3 f_{M}^{n}-4 f_{M-1}^{n}+f_{M-2}^{n}}{2 \Delta v}, & \text { for } m=M\end{cases}
$$

In summary, our numerical solutions for $f_{m}^{0}$ and $f_{m}^{1}$ are determined by (16) and (38), respectively, and the numerical solutions for $f_{m}^{n}$ with $n \geq 2$ are determined by (34). The numerical solution of $f$ obtained by the three-level Crank-Nicolson scheme has an accuracy of $O\left(\Delta \tau^{2}\right)+O\left(\Delta v^{2}\right)$
3.2. Performing Richardson's Extrapolation. To further improve the accuracy of the numerical method, we apply Richardson's extrapolation technique to $f$. We will choose $\Delta v$ proportional to $\Delta \tau$. Let $f\left(\tau_{n}, v_{m}, \Delta \tau\right)$ represent $f_{m}^{n}$ obtained by (34) with a step size $\Delta \tau$. Then,

$$
f\left(\tau_{n}, v_{m}, \Delta \tau\right)=f_{\text {exact }}(\tau, v)+C_{1} \Delta \tau^{2}+C_{2} \Delta \tau^{3}+O\left(\Delta \tau^{4}\right),
$$

where $f_{\text {exact }}$ is the exact value. We perform two computations with the step sizes $\Delta \tau$ and $\Delta \tau / 2$, respectively. Then, we have the following two equations:

$$
\begin{gathered}
f\left(\tau_{n}, v_{m}, \Delta \tau\right)=f_{\text {exact }}(\tau, v)+C_{1} \Delta \tau^{2}+C_{2} \Delta \tau^{3}+O\left(\Delta \tau^{4}\right), \\
f\left(\tau_{2 n}, v_{2 m}, \frac{\Delta \tau}{2}\right)=f_{\text {exact }}(\tau, v)+\frac{C_{1}}{2^{2}}\left(\Delta \tau^{2}\right)+\frac{C_{2}}{2^{3}} \Delta \tau^{3}+O\left(\Delta \tau^{4}\right) .
\end{gathered}
$$

From (43) and (44), we solve $f_{\text {exact }}$ and obtain an expression based on Richardson's extrapolation technique:

$$
\begin{aligned}
f_{\text {extpl. }}(n \Delta \tau, m \Delta v) & =\frac{4}{3} f\left(\tau_{2 n}, v_{2 m}, \frac{\Delta \tau}{2}\right)-\frac{1}{3} f\left(\tau_{n}, v_{m}, \Delta \tau\right) \\
& =f_{\text {exact }}+O\left(\Delta \tau^{3}\right) .
\end{aligned}
$$

After substituting $f_{\text {extpl. }}$ into (39) and (40), we obtain the expressions for $\varphi^{*}$ and $c^{*} / w$ with an accuracy of $O\left(\Delta \tau^{3}\right)$ :

$$
\begin{aligned}
& \varphi_{\text {extpl. }}^{*}=\frac{A}{\gamma}+\rho \xi \frac{\left(f_{\text {extpl. }}\right)_{v}}{f_{\text {extpl. }}}, \\
& \frac{c_{\text {extpl. }}^{*}}{w}=\left(\frac{\alpha a_{c}}{a_{w}}\right)^{(1 / \gamma)}\left(f_{\text {extpl. }}\right)^{-1},
\end{aligned}
$$

where $f_{\text {extpl. }}$ is given by $(45)$ and $\left(f_{\text {extpl. }}\right)_{v}$ is given by

$$
\left(\left(f_{\text {extpl. }}\right)_{v}\right)_{m}^{n}= \begin{cases}\frac{1}{6 \Delta v}\left[-9\left(f_{\text {extpl. }}\right)_{m}^{n}+16\left(f_{\text {extpl. }}\right)_{m+1}^{n}-8\left(f_{\text {extpl. }}\right)_{m+2}^{n}+\left(f_{\text {extpl. }}\right)_{m+4}^{n}\right], & \text { for } m \leq 1, \\ \frac{1}{6 \Delta v}\left[\left(f_{\text {extpl. }}\right)_{m-2}^{n}-4\left(f_{\text {extpl. }}\right)_{m-1}^{n}+4\left(f_{\text {extpl. }}\right)_{m+1}^{n}-\left(f_{\text {extpl. }}\right)_{m+2}^{n}\right], & \text { for } 1<m<M-1, \\ \frac{1}{6 \Delta v}\left[9\left(f_{\text {extpl. }}\right)_{m}^{n}-16\left(f_{\text {extpl. }}\right)_{m-1}^{n}+8\left(f_{\text {extpl. }}\right)_{m-2}^{n}-\left(f_{\text {extpl. }}\right)_{m-4}^{n}\right], & \text { for } m \geq M-1 .\end{cases}
$$

For the purpose of giving a quick understanding of our method, Figure 1 presents a flowchart of the algorithm for solving (19) We also summarize the procedure in words in the following for obtaining the numerical solutions of $f, \varphi^{*}$, $c^{*}, f_{\text {extpl. }}, \varphi_{\text {extpl. }}^{*}$, and $c_{\text {extpl. }}^{*}$. Here, we choose $\Delta v=\Delta \tau$.

(i) Step 1: initialize $f$ by initial condition (16), namely, $f_{m}^{0}=(1-\alpha)^{(1 / \gamma)}$, for $0 \leq m \leq M$. (ii) Step 2: initialize $f_{m}^{1}$ by (38) for $0 \leq m \leq M$.

(iii) Step 3: for $n>2$, knowing $f_{m}^{n-1}$ and $f_{m}^{n}$, for $0 \leq m \leq M, f_{m}^{n+1}$ can be determined from (34), which is in a tridiagonal form and can be easily and efficiently solved. $f_{m}^{n}$ has an accuracy of $O\left(\Delta \tau^{2}\right)$.

(iv) Step 4: to obtain $\varphi^{*}$ and $c^{*}$, we substitute $f_{m}^{n}$ from Steps 1-3 into (39) and (40). This provides the 


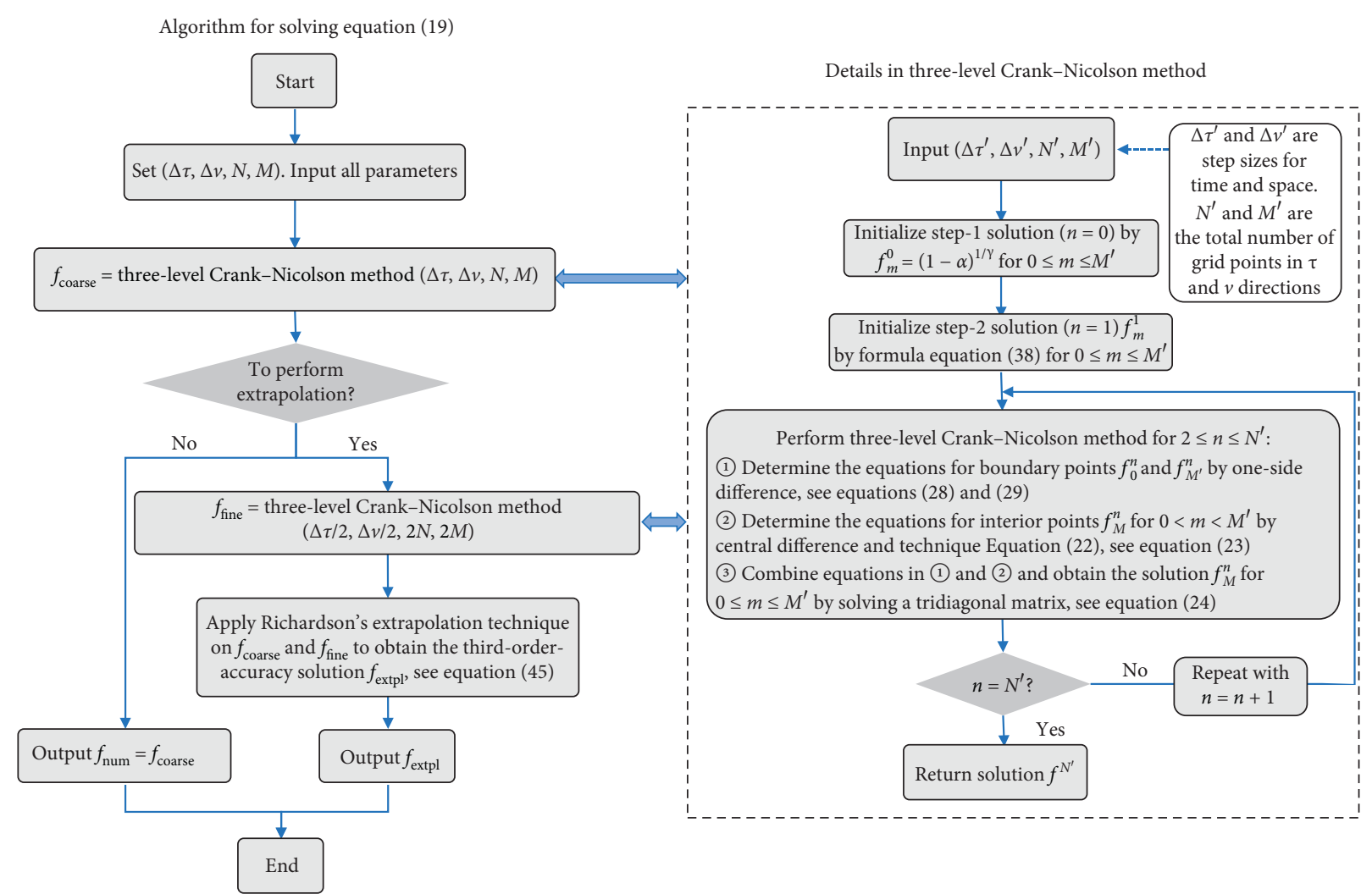

FIGURE 1: Flowchart of the algorithm.

numerical solutions for optimal strategies $\varphi_{m}^{* n}$ and $c_{m}^{* n}$ without extrapolation, which have accuracy of $O\left(\Delta \tau^{2}\right)$.

(v) Step 5: to obtain $f_{\text {extpl. }}, \varphi_{\text {extpl. }}^{*}$, and $c_{\text {extpl. }}^{*}$, we repeat Steps 1-3 with the step size $\Delta \tau / 2$ to obtain $f\left(\tau_{2 n}, v_{2 m},(\Delta \tau / 2)\right)$. Then, from (45), (46), and (47), we obtain $f_{\text {extpl. }}, \varphi_{\text {extpl. }}^{*}$, and $c_{\text {extpl. }}^{*}$, all of which have accuracy of $O\left(\Delta \tau^{3}\right)$.

In the next section, we will verify the accuracy of the numerical solutions without extrapolation and those with extrapolation.

\section{Validation Study of the Numerical Method}

Equation (19) is an inhomogeneous equation. However, since the inhomogeneous term $\alpha^{(1 / \gamma)} a_{7}$ affects neither the stability nor the accuracy of the three-level Crank-Nicolson method, it is sufficient to conduct validation studies for the corresponding homogeneous equation, namely, for the case of $\alpha=0$. Let $\hat{f}$ be the solution of the homogeneous equation of (19), namely, the case of $\alpha=0$. Then, $\widehat{f}$ satisfies

$$
\begin{aligned}
& -(\widehat{f})_{\tau}+a_{1} v(\widehat{f})_{v v}+\left(a_{2} v+a_{3}\right)(\widehat{f})_{v}+a_{4} v \frac{(\widehat{f})_{v}^{2}}{\widehat{f}} \\
& +\left(a_{5} v+a_{6}\right) \hat{f}=0,
\end{aligned}
$$

with the initial condition $\hat{f}(0, v)=1$. Following the procedure outlined in [10], the exact solution for $\widehat{f}$ can be obtained. After expressing $\hat{f}(\tau, v)$ as

$$
\widehat{f}(\tau, v)=e^{h_{1}(\tau) v+h_{2}(\tau)},
$$

from (49), $h_{1}(\tau)$ and $h_{2}(\tau)$ are governed by

$$
\begin{array}{rlrl}
h_{1}^{\prime}(\tau)-\left(a_{1}+a_{4}\right) h_{1}(\tau)^{2}-a_{2} h_{1}(\tau)-a_{5}=0, & & \text { with } h_{1}(0)=0, \\
h_{2}^{\prime}(\tau)-a_{3} h_{1}(\tau)-a_{6}=0, & \text { with } h_{2}(0)=0,
\end{array}
$$

and the solutions are 


$$
\begin{aligned}
& h_{1}(\tau)= \begin{cases}\frac{2 a_{5}\left(e^{\sqrt{\Delta} \tau}-1\right)}{a_{2}+\sqrt{\Delta}-\left(a_{2}-\sqrt{\Delta}\right) e^{\sqrt{\Delta} \tau},} & \text { for } \Delta>0, \\
-\frac{2 a_{5} \tau}{a_{2} \tau-2}, & \text { for } \Delta=0, \\
\frac{2 a_{5}}{\sqrt{-\Delta}\left(\cot (\sqrt{-\Delta} \tau / 2)-\left(a_{2} / \sqrt{-\Delta}\right)\right)}, & \text { for } \Delta<0,\end{cases} \\
& h_{2}(\tau)= \begin{cases}-\frac{a_{3}}{a_{8}} \log \left|\frac{\left(a_{2}+\sqrt{\Delta}\right) e^{-\sqrt{\Delta} \tau}-a_{2}+\sqrt{\Delta}}{2 \sqrt{\Delta}}\right|+\left(a_{6}-\frac{a_{3} \sqrt{\Delta}}{2 a_{8}}-\frac{a_{2} a_{3}}{2 a_{8}}\right) \tau, & \text { for } \Delta>0, \\
-\frac{a_{3}}{a_{8}} \log \left|1-\frac{a_{2} \tau}{2}\right|+\left(a_{6}-\frac{a_{2} a_{3}}{2 a_{8}}\right) \tau, & \text { for } \Delta=0, \\
-\frac{a_{3}}{a_{8}} \log \left|\cos \left(\frac{\sqrt{-\Delta} \tau}{2}\right)-\frac{a_{2}}{\sqrt{-\Delta}} \sin \left(\frac{\sqrt{-\Delta} \tau}{2}\right)\right|+\left(a_{6}-\frac{a_{2} a_{3}}{2 a_{8}}\right) \tau, & \text { for } \Delta<0,\end{cases}
\end{aligned}
$$

where $a_{8}=a_{1}+a_{4}$ and $\Delta=a_{2}^{2}-4 a_{5} a_{8}$. From (17) and (18), we obtain the exact solutions of optimal strategies $\widehat{\varphi}^{*}$ and $\widehat{c}^{*}$ for the case of $\alpha=0$ :

$$
\begin{aligned}
& \widehat{\varphi}_{\text {exact }}^{*}=\frac{A}{\gamma}+\rho \xi h_{1}(\tau), \\
& \frac{\widehat{c}_{\text {exact }}^{*}}{w}=0,
\end{aligned}
$$

where $h_{1}(\tau)$ is given by $(52)$.

The exact solutions $\widehat{f}_{\text {exact }}, \widehat{\varphi}_{\text {exact }}^{*}$, and $\widehat{c}_{\text {exact }}^{*}$ for the case of $\alpha=0$ given by (50), (54), and (55) offer a benchmark for testing the accuracy of our numerical solutions. We show that our numerical solutions are accurate and efficient for $\alpha=0$. Since neither the inhomogeneous term $\alpha^{(1 / \gamma)} a_{7}$ nor the constant initial condition $(1-\alpha)^{(1 / \gamma)}$ affects the stability or the accuracy of a numerical method, the accuracy and the stability of the method remain valid for $\alpha \neq 0$. The numerical results for $\alpha \neq 0$ are presented at the end of this section.

4.1. Numerical Validation. To set parameters for numerical validation, we use the estimation values of the parameters $\kappa, \theta, \xi, \rho, A, \beta$, and $\gamma$ given in $[4,15,22,23]$ and the historical records of $r$. These values are listed in Table 1 .

We note that since $a_{w}$ determines the wealth scale and $a_{w} / a_{c}$ determines the temporal scale, without loss of generality, we choose $a_{c}=a_{w}=1$ in this study.

For the range of the state variables $t$ and $v$, we consider $T \leq 100$. Based on the historical records of the Chicago Board Options Exchange Volatility Index, a popular measure of the implied volatility of S\&P 500 index options, we examine the numerical solutions for the instantaneous volatility $\sigma_{t}=\sqrt{v_{t}}$ in the interval $[0.1,0.8]$ (to eliminate possible influence from the numerical boundary, the authors choose $v_{\max }=2$ in their numerical computations). Since wealth $w$ does not appear in (19), (17), and (18), its value is irrelevant in our study.
In Table 2, we show the comparison between $\hat{f}_{\text {exact }}$, $\widehat{f}_{\text {num. }}$, and $\hat{f}_{\text {extpl. }} \cdot \hat{f}_{\text {exact }}$ is the exact solution of (49). When $\alpha=0, \widehat{f}_{\text {num. }}$ given by (34) is the numerical solution of (49) without performing Richardson's extrapolation and $\widehat{f}_{\text {extpl. }}$ given by (45) is the numerical solution of (49) after performing Richardson's extrapolation. The relative errors in $\widehat{f}_{\text {num. }}$ and $\widehat{f}_{\text {extpl., }}$ namely, $\left|\left(\widehat{f}_{\text {num. }}-\widehat{f}_{\text {exact }}\right) / \widehat{f}_{\text {exact }}\right|$ and $\left|\left(\widehat{f}_{\text {extpl. }}-\widehat{f}_{\text {exact }}\right) / \widehat{f}_{\text {exact }}\right|$, are shown in the last two columns of Table 2 .

In Table 3, we show the comparison between the exact solution $\widehat{\varphi}_{\text {exact }}^{*}$ given by (54), the numerical solution $\widehat{\varphi}_{\text {num. }}^{*}$ determined from (39) without performing Richardson's extrapolation, and $\widehat{\varphi}_{\text {extpl. }}^{*}$ determined from (46) after performing Richardson's extrapolation. The relative errors in $\widehat{\varphi}_{\text {num. }}^{*}$ and $\widehat{\varphi}_{\text {extpl. }}^{*}$, namely, $\left|\left(\widehat{\varphi}_{\text {num. }}^{*}-\widehat{\varphi}_{\text {exact }}^{*}\right) / \widehat{\varphi}_{\text {exact }}^{*}\right|$ and $\left|\left(\widehat{\varphi}_{\text {extpl. }}^{*}-\widehat{\varphi}_{\text {exact }}^{*}\right) / \widehat{\varphi}_{\text {exact }}^{*}\right|$, are shown in the last two columns of Table 3.

There is no error in $c^{*}$ since both numerical and theoretical values of $c^{*}$ are zero.

In Table 4, we show the global relative errors and the computational times of $\widehat{f}_{\text {num. }}$ and $\widehat{f}_{\text {extpl. }}$ for different values of $\Delta \tau$ and $\Delta v$. The global relative error in $\widehat{f}_{\text {num. }}$ is defined as the maximum of the local relative errors between $\widehat{f}_{\text {exact }}$ and $\widehat{f}_{\text {num. }}$ in the domain $0 \leq \tau \leq 100$ and $0 \leq \sqrt{v}=\sigma \leq 0.8$. The global relative error in $\widehat{f}_{\text {extpl. }}$ is defined in the same way.

Table 4 confirms that our numerical solutions $\widehat{f}_{\text {num. }}$ and $\widehat{\varphi}_{\text {num. }}^{*}$ have accuracy at orders of $\Delta \tau^{2}$ and that $\widehat{f}_{\text {extpl. }}$ and $\widehat{\varphi}_{\text {extpl. }}^{*}$ have accuracy at orders of $\Delta \tau^{3}$. Therefore, the extrapolation technique does improve the accuracy. For the same order of accuracy, the application of Richardson's extrapolation technique significantly saves the computational time.

4.2. Extensive Sets of Parameters. In this section, some extensive sets of parameters are used to further validate the proposed method. Since this system contains several 
TABLE 1: Values for the parameters $\kappa, \theta, \xi, \rho, A, r, \beta, \gamma, a_{c}$, and $a_{w}$.

\begin{tabular}{lcccccccccc}
\hline Parameter & $\kappa$ & $\theta$ & $\xi$ & $\rho$ & $A$ & $r$ & $\beta$ & $\gamma$ & $a_{c}$ & $a_{w}$ \\
\hline Value & 1.6048 & 0.0464 & 0.3796 & $-76.70 \%$ & 1.55 & $1 \%$ & 0.06 & 2 & 1 & 1 \\
\hline
\end{tabular}

TABLE 2: Comparison between the exact solution $\hat{f}_{\text {exact }}$, the numerical solution without Richardson's extrapolation $\hat{f}_{\text {num. }}$, and the numerical solution after Richardson's extrapolation $\widehat{f}_{\text {extpl. }}$. The relative errors in numerical solutions are shown in the last two columns. Here, the numerical solutions are obtained with $\Delta \tau=\Delta v=0.01$.

\begin{tabular}{|c|c|c|c|c|c|c|}
\hline$\tau$ & $\sigma=\sqrt{v}$ & $\widehat{f}_{\text {exact }}$ & $\widehat{f}_{\text {num. }}$ & $\widehat{f}_{\text {extpl. }}$ & $\left|\left(\widehat{f}_{\text {num. }}-\widehat{f}_{\text {exact }}\right) / \widehat{f}_{\text {exact }}\right|$ & $\left|\left(\widehat{f}_{\text {extpl. }}-\widehat{f}_{\text {exact }}\right) / \widehat{f}_{\text {exact }}\right|$ \\
\hline 0.1 & 0.1 & 0.9961202112 & 0.9961202904 & 0.9961202112 & $7.9 \times 10^{-8}$ & $2.1 \times 10^{-12}$ \\
\hline 0.1 & 0.4 & 0.9919378844 & 0.9919376809 & 0.9919378844 & $2.1 \times 10^{-7}$ & $6.2 \times 10^{-12}$ \\
\hline 0.1 & 0.8 & 0.9786720788 & 0.9786706845 & 0.9786720789 & $1.4 \times 10^{-6}$ & $8.1 \times 10^{-11}$ \\
\hline 1 & 0.1 & 0.9569365332 & 0.9569367514 & 0.9569365330 & $2.3 \times 10^{-7}$ & $1.3 \times 10^{-10}$ \\
\hline 1 & 0.4 & 0.9339516557 & 0.9339511052 & 0.9339516557 & $5.9 \times 10^{-7}$ & $1.4 \times 10^{-11}$ \\
\hline 1 & 0.8 & 0.8640451414 & 0.8640421623 & 0.8640451415 & $3.4 \times 10^{-6}$ & $8.9 \times 10^{-11}$ \\
\hline 10 & 0.1 & 0.6062531863 & 0.6062531702 & 0.6062531839 & $2.6 \times 10^{-8}$ & $4.0 \times 10^{-9}$ \\
\hline 10 & 0.4 & 0.5870696178 & 0.5870696136 & 0.5870696159 & $7.1 \times 10^{-9}$ & $3.2 \times 10^{-9}$ \\
\hline 10 & 0.8 & 0.5296676303 & 0.5296676662 & 0.5296676288 & $6.8 \times 10^{-8}$ & $2.7 \times 10^{-9}$ \\
\hline 100 & 0.1 & 0.0061763000 & 0.0061762986 & 0.0061762997 & $2.2 \times 10^{-7}$ & $4.2 \times 10^{-8}$ \\
\hline 100 & 0.4 & 0.0059808642 & 0.0059808630 & 0.0059808640 & $2.0 \times 10^{-7}$ & $4.1 \times 10^{-8}$ \\
\hline 100 & 0.8 & 0.0053960721 & 0.0053960714 & 0.0053960718 & $1.3 \times 10^{-7}$ & $4.0 \times 10^{-8}$ \\
\hline
\end{tabular}

TABLE 3: Comparison between the exact solution $\widehat{\varphi}_{\text {exact }}^{*}$, the numerical solution without Richardson's extrapolation $\widehat{\varphi}_{\text {num. }}^{*}$, and the numerical solution after Richardson's extrapolation $\widehat{\varphi}_{\text {extpl. }}^{*}$ The relative errors in numerical solutions are shown in the last two columns. Here, the numerical solutions are obtained with $\Delta \tau=\Delta v=0.01$.

\begin{tabular}{|c|c|c|c|c|c|c|}
\hline$\tau$ & $\sigma=\sqrt{v}$ & $\widehat{\varphi}_{\text {exact }}^{*}$ & $\widehat{\varphi}_{\text {num. }}^{*}$ & $\widehat{\varphi}_{\text {extpl. }}^{*}$ & $\left|\left(\widehat{\varphi}_{\text {num. }}^{*}-\widehat{\varphi}_{\text {exact }}^{*}\right) / \widehat{\varphi}_{\text {exact }}^{*}\right|$ & $\left|\left(\widehat{\varphi}_{\text {extpl. }}^{*}-\widehat{\varphi}_{\text {exact }}^{*}\right) / \widehat{\varphi}_{\text {exact }}^{*}\right|$ \\
\hline 0.1 & 0.1 & 0.7831667609 & 0.7831672706 & 0.7831667609 & $6.5 \times 10^{-7}$ & $1.5 \times 10^{-11}$ \\
\hline 0.1 & 0.4 & 0.7831667609 & 0.7831673568 & 0.7831667609 & $7.6 \times 10^{-7}$ & $2.8 \times 10^{-11}$ \\
\hline 0.1 & 0.8 & 0.7831667609 & 0.7831676487 & 0.7831667608 & $1.1 \times 10^{-6}$ & $9.1 \times 10^{-11}$ \\
\hline 1 & 0.1 & 0.8221908761 & 0.8221924687 & 0.8221908741 & $1.9 \times 10^{-6}$ & $2.4 \times 10^{-9}$ \\
\hline 1 & 0.4 & 0.8221908761 & 0.8221925174 & 0.8221908760 & $2.0 \times 10^{-6}$ & $6.6 \times 10^{-11}$ \\
\hline 1 & 0.8 & 0.8221908761 & 0.8221927448 & 0.8221908760 & $2.3 \times 10^{-6}$ & $5.8 \times 10^{-11}$ \\
\hline 10 & 0.1 & 0.8374121549 & 0.8374122233 & 0.8374121478 & $8.2 \times 10^{-8}$ & $8.6 \times 10^{-9}$ \\
\hline 10 & 0.4 & 0.8374121549 & 0.8374121589 & 0.8374121544 & $4.8 \times 10^{-9}$ & $6.4 \times 10^{-10}$ \\
\hline 10 & 0.8 & 0.8374121549 & 0.8374121565 & 0.8374121548 & $1.9 \times 10^{-9}$ & $1.6 \times 10^{-10}$ \\
\hline 100 & 0.1 & 0.8374121969 & 0.8374122652 & 0.8374121897 & $8.2 \times 10^{-8}$ & $8.6 \times 10^{-9}$ \\
\hline 100 & 0.4 & 0.8374121969 & 0.8374122009 & 0.8374121964 & $4.7 \times 10^{-9}$ & $6.4 \times 10^{-10}$ \\
\hline 100 & 0.8 & 0.8374121969 & 0.8374121985 & 0.8374121968 & $1.9 \times 10^{-9}$ & $1.6 \times 10^{-10}$ \\
\hline
\end{tabular}

TABLE 4: Maximum relative errors and computational times of $\widehat{f}_{\text {num. }}$ and $\widehat{f}_{\text {extpl. }}$ in the domain $0 \leq \tau \leq 100$ and $0 \leq \sqrt{v}=\sigma \leq 0.8$.

\begin{tabular}{|c|c|c|c|c|c|}
\hline \multicolumn{2}{|c|}{ Step size } & \multicolumn{2}{|c|}{$\widehat{\widehat{f}}_{\text {num. }}$} & \multicolumn{2}{|c|}{$\widehat{f}_{\text {extpl. }}$} \\
\hline$\Delta \tau$ & $\Delta v$ & Max. rel. err. & Comp. time & Max. rel. err. & Comp. time \\
\hline 0.02 & 0.02 & $1.5 \times 10^{-5}$ & 0.39 & $3.3 \times 10^{-7}$ & 0.84 \\
\hline 0.01 & 0.01 & $3.8 \times 10^{-6}$ & 1.52 & $4.2 \times 10^{-8}$ & 3.31 \\
\hline 0.005 & 0.005 & $9.6 \times 10^{-7}$ & 6.02 & $5.2 \times 10^{-9}$ & 13.18 \\
\hline 0.0025 & 0.0025 & $2.4 \times 10^{-7}$ & 24.02 & $6.3 \times 10^{-10}$ & 52.54 \\
\hline
\end{tabular}

parameters, we vary only one of the parameters at a time and keep other parameters at their benchmark values as shown in Table 1. Extensive sets are given in Table 5.

By choosing the step size $\Delta \tau=0.01$ and $\Delta v=0.01$, the proposed algorithm is conducted, and the relative errors are recorded both before and after performing the extrapolation technique. In Tables 6 and 7, we show the maximum relative errors of the proposed algorithm before and after performing the extrapolation technique, respectively, in the domain $0 \leq \tau \leq 100$ and $0 \leq \sqrt{v}=\sigma \leq 0.8$. It can be found that the extrapolation technique does improve one-order accuracy for the ten sets of parameters in Table 5.

By taking half of the step size above, namely, choosing $\Delta \tau=0.005$ and $\Delta v=0.005$, the proposed algorithm is 
TABLE 5: Values for the parameters $\kappa, \theta, \xi, \rho, A, r, \beta, \gamma, a_{c}$, and $a_{w}$.

\begin{tabular}{lcccccccccc}
\hline Parameter & $\kappa$ & $\theta$ & $\xi$ & $\rho$ & $A$ & $r$ & $\beta$ & $\gamma$ & $a_{c}$ & $a_{w}$ \\
\hline Set 1 & 2.5 & 0.0464 & 0.3796 & $-76.70 \%$ & 1.55 & $1 \%$ & 0.06 & 2 & 1 \\
Set 2 & 1.6048 & 0.08 & 0.3796 & $-76.70 \%$ & 1.55 & $1 \%$ & 0.06 & 2 & 1 \\
Set 3 & 1.6048 & 0.0464 & 0.6 & $-76.70 \%$ & 1.55 & $1 \%$ & 0.06 & 2 & 1 \\
Set 4 & 1.6048 & 0.0464 & 0.3796 & $-50 \%$ & 1.55 & $1 \%$ & 0.06 & 2 & 1 \\
Set 5 & 1.6048 & 0.0464 & 0.3796 & $-76.70 \%$ & 1.25 & $1 \%$ & 0.06 & 2 & 1 \\
Set 6 & 1.6048 & 0.0464 & 0.3796 & $-76.70 \%$ & 1.55 & $5 \%$ & 0.06 & 2 & 1 \\
Set 7 & 1.6048 & 0.0464 & 0.3796 & $-76.70 \%$ & 1.55 & $1 \%$ & 0.15 & 2 & 1 \\
Set 8 & 1.6048 & 0.0464 & 0.3796 & $-76.70 \%$ & 1.55 & $1 \%$ & 0.06 & 5 & 1 \\
Set 9 & 1.6048 & 0.0464 & 0.3796 & $-76.70 \%$ & 1.55 & $1 \%$ & 0.06 & 2 & 2 \\
Set 10 & 1.6048 & 0.0464 & 0.3796 & $-76.70 \%$ & 1.55 & $1 \%$ & 0.06 & 2 & 1 \\
\hline
\end{tabular}

TABle 6: Maximum relative error for $\hat{f}_{\text {num. }}$ and $\widehat{\varphi}_{\text {num. }}$ with $\Delta \tau=0.01$ and $\Delta v=0.01$.

\begin{tabular}{lcc}
\hline Set & $\hat{f}_{\text {num. }}$ & Maximum relative error \\
\hline Set 1 & $5.8 \times 10^{-6}$ & $3.9 \times 10^{-6}$ \\
Set 2 & $3.6 \times 10^{-6}$ & $2.7 \times 10^{-6}$ \\
Set 3 & $3.6 \times 10^{-6}$ & $3.9 \times 10^{-6}$ \\
Set 4 & $4.0 \times 10^{-6}$ & $1.8 \times 10^{-6}$ \\
Set 5 & $2.4 \times 10^{-6}$ & $2.0 \times 10^{-6}$ \\
Set 6 & $4.0 \times 10^{-6}$ & $2.8 \times 10^{-6}$ \\
Set 7 & $4.2 \times 10^{-6}$ & $2.9 \times 10^{-6}$ \\
Set 8 & $2.1 \times 10^{-6}$ & $1.4 \times 10^{-6}$ \\
Set 9 & $3.8 \times 10^{-6}$ & $2.7 \times 10^{-6}$ \\
Set 10 & $3.8 \times 10^{-6}$ & $2.7 \times 10^{-6}$ \\
\hline
\end{tabular}

TABLE 7: Maximum relative error for $\widehat{f}_{\text {extpl. }}$ and $\widehat{\varphi}_{\text {extpl. }}$ with $\Delta \tau=0.01$ and $\Delta v=0.01$.

\begin{tabular}{lcc}
\hline Set & $\hat{f}_{\text {extpl. }}$ & Maximum relative error \\
\hline Set 1 & $1.2 \times 10^{-7}$ & $\widehat{\varphi}_{\text {extpl. }}$ \\
Set 2 & $5.0 \times 10^{-8}$ & $9.3 \times 10^{-8}$ \\
Set 3 & $9.5 \times 10^{-8}$ & $4.3 \times 10^{-8}$ \\
Set 4 & $5.9 \times 10^{-8}$ & $5.9 \times 10^{-8}$ \\
Set 5 & $3.3 \times 10^{-8}$ & $3.1 \times 10^{-8}$ \\
Set 6 & $5.6 \times 10^{-8}$ & $3.1 \times 10^{-8}$ \\
Set 7 & $5.8 \times 10^{-8}$ & $4.5 \times 10^{-8}$ \\
Set 8 & $2.5 \times 10^{-8}$ & $4.7 \times 10^{-8}$ \\
Set 9 & $5.4 \times 10^{-8}$ & $2.02 \times 10^{-8}$ \\
Set 10 & $5.4 \times 10^{-8}$ & $4.4 \times 10^{-8}$ \\
\end{tabular}

TABLE 8: Maximum relative error for $\widehat{f}_{\text {num. }}$ and $\widehat{\varphi}_{\text {num. }}$ with $\Delta \tau=0.005$ and $\Delta v=0.005$.

\begin{tabular}{lcr}
\hline Set & \multicolumn{1}{c}{ Maximum relative error } \\
\hline Set 1 & $\widehat{f}_{\text {num. }}$ & $9.7 \times 10^{-7}$ \\
Set 2 & $1.4 \times 10^{-6}$ & $6.7 \times 10^{-7}$ \\
Set 3 & $9.1 \times 10^{-7}$ & $9.8 \times 10^{-7}$ \\
Set 4 & $9.0 \times 10^{-7}$ & $4.6 \times 10^{-7}$ \\
Set 5 & $1.0 \times 10^{-6}$ & $5.0 \times 10^{-7}$ \\
Set 6 & $6.0 \times 10^{-7}$ & $6.9 \times 10^{-7}$ \\
Set 7 & $10.0 \times 10^{-7}$ & $7.2 \times 10^{-7}$ \\
Set 8 & $1.0 \times 10^{-6}$ & $3.5 \times 10^{-7}$ \\
Set 9 & $5.1 \times 10^{-7}$ & $6.7 \times 10^{-7}$ \\
Set 10 & $9.6 \times 10^{-7}$ & $6.7 \times 10^{-7}$ \\
\hline
\end{tabular}


TABLE 9: Maximum relative error for $\widehat{f}_{\text {extpl. }}$ and $\widehat{\varphi}_{\text {extpl. }}$ with $\Delta \tau=0.005$ and $\Delta v=0.005$.

\begin{tabular}{lcr}
\hline Set & \multicolumn{1}{c}{ Maximum relative error } \\
\hline Set 1 & $\hat{f}_{\text {extpl. }}$ & $1.2 \times 10^{-8}$ \\
Set 2 & $1.6 \times 10^{-8}$ & $7.5 \times 10^{-9}$ \\
Set 3 & $6.4 \times 10^{-9}$ & $7.6 \times 10^{-9}$ \\
Set 4 & $2.1 \times 10^{-8}$ & $4.0 \times 10^{-9}$ \\
Set 5 & $7.6 \times 10^{-9}$ & $4.0 \times 10^{-9}$ \\
Set 6 & $4.2 \times 10^{-9}$ & $5.8 \times 10^{-9}$ \\
Set 7 & $7.1 \times 10^{-9}$ & $6.0 \times 10^{-9}$ \\
Set 8 & $7.4 \times 10^{-9}$ & $2.5 \times 10^{-9}$ \\
Set 9 & $3.2 \times 10^{-9}$ & $5.6 \times 10^{-9}$ \\
Set 10 & $6.9 \times 10^{-9}$ & $5.6 \times 10^{-9}$ \\
\hline
\end{tabular}

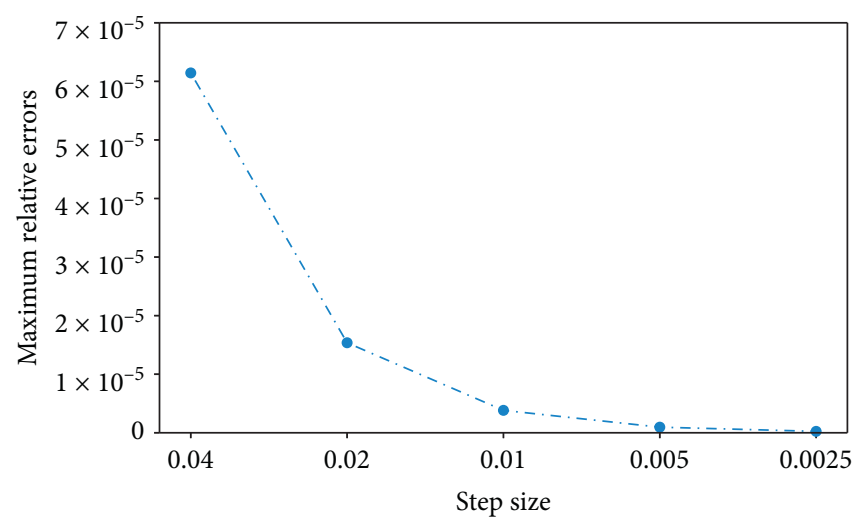

Figure 2: Maximum relative errors for decreasing step size.

TABLE 10: Maximum relative errors and convergence orders of $\widehat{f}_{\text {num. }}$ in the domain $0 \leq \tau \leq 100$ and $0 \leq \sqrt{v}=\sigma \leq 0.8$.

\begin{tabular}{|c|c|c|c|}
\hline \multicolumn{2}{|c|}{ Step size } & \multirow{2}{*}{ Maximum relative errors } & \multirow{2}{*}{ Convergence order } \\
\hline$\Delta \tau$ & $\Delta v$ & & \\
\hline 0.02 & 0.02 & $1.53 \times 10^{-5}$ & 2.0 \\
\hline 0.01 & 0.01 & $3.83 \times 10^{-6}$ & 2.0 \\
\hline 0.005 & 0.005 & $9.58 \times 10^{-7}$ & 2.0 \\
\hline 0.0025 & 0.0025 & $2.40 \times 10^{-7}$ & 2.0 \\
\hline
\end{tabular}

conducted, and the relative errors are recorded both before and after performing the extrapolation technique. In $\mathrm{Ta}$ bles 8 and 9 , we show the maximum relative errors of the proposed algorithm before and after performing the extrapolation technique, respectively, in the domain $0 \leq \tau \leq 100$ and $0 \leq \sqrt{v}=\sigma \leq 0.8$. Tables $6-9$ show that the proposed algorithm before and after performing the extrapolation technique has second-order accuracy and thirdorder accuracy, respectively, for the extensive sets of parameters.

4.3. Evidence for Stability and Convergence. To provide the evidence for stability, we calculate the maximum relative errors of $\widehat{f}_{\text {num. }}$ for $\Delta \tau=\Delta v=0.04,0.02,0.01,0.005,0.0025$, respectively. The results are shown in Figure 2. One can see that, as the step size goes to zero, the maximum relative errors tend to zero, which numerically verifies the stability of the proposed algorithm.

To provide the evidence for convergence, let $e(\Delta \tau, \Delta v)$ be the maximum relative error; then, the convergence order is given by

$$
\text { convergence order }=\log _{2}\left(\frac{e(\Delta \tau, \Delta v)}{e((\Delta \tau / 2),(\Delta v / 2))}\right) \text {. }
$$

In Table 10, the maximum relative errors and convergence orders of $\widehat{f}_{\text {num. }}$ for different values of $\Delta \tau$ and $\Delta v$ are given. It shows that the convergence orders are always equal to 2.0 as $\Delta \tau$ and $\Delta v$ go to zero, which numerically verifies the convergence of the proposed algorithm. 
TABLE 11: Numerical solutions for $f, \varphi^{*}$, and $c^{*} / w$ after performing Richardson's extrapolation. Here, $\alpha=0.1$ and $\Delta \tau=\Delta v=0.001$. Other parameter values are the same as those in Table 1.

\begin{tabular}{lcccc}
\hline$\tau$ & $\sigma=\sqrt{v}$ & $f_{\text {extpl. }}$ & $\varphi_{\text {extpl. }}^{*}$ & $c_{\text {extpl. }}^{*} / w$ \\
\hline 0.1 & 0.1 & 0.976564446 & 0.783037744 & 0.323816587 \\
0.1 & 0.4 & 0.972528955 & 0.783037394 & 0.325160258 \\
0.1 & 0.8 & 0.959728271 & 0.783036268 & 0.329497187 \\
1 & 0.1 & 1.217443571 & 0.817505616 & 0.259747370 \\
1 & 0.4 & 1.191090583 & 0.817448428 & 0.265494305 \\
1 & 0.8 & 1.110757357 & 0.817260409 & 0.284695630 \\
10 & 0.1 & 3.073495770 & 0.833010306 & 0.102888629 \\
10 & 0.4 & 2.983046823 & 0.832947671 & 0.106008315 \\
10 & 0.8 & 2.711715965 & 0.832739549 & 0.116615372 \\
100 & 0.1 & 6.227692985 & 0.835240145 & 0.050777674 \\
100 & 0.4 & 6.037435062 & 0.835206694 & 0.052377833 \\
100 & 0.8 & 5.467451073 & 0.835095101 & 0.057838243 \\
\hline
\end{tabular}

TABLE 12: Numerical solutions for $f, \varphi^{*}$, and $c^{*} / w$ after performing Richardson's extrapolation. Here, $\alpha=0.5$ and $\Delta \tau=\Delta v=0.001$. Other parameter values are the same as those in Table 1.

\begin{tabular}{lcccc}
\hline$\tau$ & $\sigma=\sqrt{v}$ & $f_{\text {extpl. }}$ & $\varphi_{\text {extpl. }}^{*}$ & $c_{\text {extpl. }}^{*} / w$ \\
\hline 0.1 & 0.1 & 0.774937774 & 0.782803210 & 0.912469110 \\
0.1 & 0.4 & 0.771828844 & 0.782802269 & 0.916144540 \\
0.1 & 0.8 & 0.761966328 & 0.782799238 & 0.928002663 \\
1 & 0.1 & 1.368974100 & 0.812872895 & 0.516523126 \\
1 & 0.4 & 1.342553293 & 0.812781707 & 0.526688054 \\
1 & 0.8 & 1.261787768 & 0.812484452 & 0.560400726 \\
10 & 0.1 & 6.015181176 & 0.832382642 & 0.117553696 \\
10 & 0.4 & 5.840061390 & 0.832312731 & 0.121078656 \\
10 & 0.8 & 5.314527422 & 0.832080689 & 0.133051676 \\
100 & 0.1 & 13.916810422 & 0.835238781 & 0.050809543 \\
100 & 0.4 & 13.491657157 & 0.835205311 & 0.052410669 \\
100 & 0.8 & 12.217961221 & 0.835093653 & 0.057874368 \\
\hline
\end{tabular}

TABLE 13: Numerical solutions for $f, \varphi^{*}$, and $c^{*} / w$ after performing Richardson's extrapolation. Here, $\alpha=0.9$ and $\Delta \tau=\Delta v=0.001$. Other parameter values are the same as those in Table 1.

\begin{tabular}{lcccc}
\hline$\tau$ & $\sigma=\sqrt{v}$ & $f_{\text {extpl. }}$ & $\varphi_{\text {extpl. }}^{*}$ & $c_{\text {extpl. }}^{*} / w$ \\
\hline 0.1 & 0.1 & 0.409686387 & 0.782244153 & 2.315632955 \\
0.1 & 0.4 & 0.408160455 & 0.782242030 & 2.324290085 \\
0.1 & 0.8 & 0.403318534 & 0.782235205 & 2.352193658 \\
1 & 0.1 & 1.231451909 & 0.808291630 & 0.770377869 \\
1 & 0.4 & 1.210542696 & 0.808188721 & 0.783684294 \\
1 & 0.8 & 1.146400499 & 0.807855696 & 0.827532175 \\
10 & 0.1 & 7.686789813 & 0.832131657 & 0.123417359 \\
10 & 0.4 & 7.463974966 & 0.832058953 & 0.127101618 \\
10 & 0.8 & 6.795202083 & 0.831817743 & 0.139610756 \\
100 & 0.1 & 18.667454349 & 0.835238326 & 0.050820175 \\
100 & 0.4 & 18.097174990 & 0.835204850 & 0.052421624 \\
100 & 0.8 & 16.388702420 & 0.835093170 & 0.057886419 \\
\hline
\end{tabular}

4.4. Numerical Results for $\alpha \neq 0$. We have confirmed the accuracy of our numerical solutions for $\alpha=0$. This guarantees that our numerical solution $f_{\text {extpl. }}$ for $\alpha \neq 0$ will also have an accuracy of $O\left(\Delta \tau^{3}\right)$. In Tables $11-13$, we present the numerical solutions of $f_{\text {extpl. }}, \varphi_{\text {extpl. }}^{*}$, and $c_{\text {extpl. }}^{*} / w$ for $\alpha=0.1,0.5$, and 0.9 with step sizes $\Delta \tau=\Delta v=0.001$. In
Tables 14 and 15, we show the results for $\gamma=1$ and 10 with $\alpha=0.5$ and $\Delta \tau=\Delta v=0.001$. All other parameter values in Tables 11-15 are the same as the ones in Table 1. All digits shown in Tables 11-15 are exact, in the sense that they do not change when we further refine the values of $\Delta \tau$ and $\Delta v$. Therefore, we have provided the first set of exact numerical 
TABLE 14: Numerical solutions for $f, \varphi^{*}$, and $c^{*} / w$ after performing Richardson's extrapolation. Here, $\alpha=0.5, \gamma=1$, and $\Delta \tau=\Delta v=0.001$. Other parameter values are the same as those in Table 1.

\begin{tabular}{lcccc}
\hline$\tau$ & $\sigma=\sqrt{v}$ & $f_{\text {extpl. }}$ & $\varphi_{\text {extpl. }}^{*}$ & $c_{\text {extpl. }}^{*} / w$ \\
\hline 0.1 & 0.1 & 0.546859282 & 1.550000000 & 0.914311994 \\
0.1 & 0.4 & 0.546859282 & 1.550000000 & 0.914311994 \\
0.1 & 0.8 & 0.546859282 & 1.550000000 & 0.914311994 \\
1 & 0.1 & 0.956177820 & 1.550000000 & 0.522915288 \\
1 & 0.4 & 0.956177820 & 1.550000000 & 0.522915288 \\
1 & 0.8 & 0.956177820 & 1.550000000 & 0.522915288 \\
10 & 0.1 & 4.034308851 & 1.550000000 & 0.123936966 \\
10 & 0.4 & 4.034308851 & 1.550000000 & 0.123936966 \\
10 & 0.8 & 4.034308851 & 1.550000000 & 0.123936966 \\
100 & 0.1 & 8.313916441 & 1.550000000 & 0.060140128 \\
100 & 0.4 & 8.313916441 & 1.550000000 & 0.060140128 \\
100 & 0.8 & 8.313916441 & 1.550000000 & 0.060140128 \\
\hline
\end{tabular}

TABLE 15: Numerical solutions for $f, \varphi^{*}$, and $c^{*} / w$ after performing Richardson's extrapolation. Here, $\alpha=0.5, \gamma=10$, and $\Delta \tau=\Delta v=0.001$. Other parameter values are the same as those in Table 1.

\begin{tabular}{lcccc}
\hline$\tau$ & $\sigma=\sqrt{v}$ & $f_{\text {extpl. }}$ & $\varphi_{\text {extpl. }}^{*}$ & $c_{\text {extpl. }}^{*} / w$ \\
\hline 0.1 & 0.1 & 1.024730849 & 0.157833805 & 0.910515178 \\
0.1 & 0.4 & 1.023235912 & 0.157833681 & 0.911845432 \\
0.1 & 0.8 & 1.018467192 & 0.157833282 & 0.916114922 \\
1 & 0.1 & 1.840789858 & 0.169544364 & 0.506865565 \\
1 & 0.4 & 1.827054636 & 0.169530453 & 0.510676021 \\
1 & 0.8 & 1.783873146 & 0.169485620 & 0.523037747 \\
10 & 0.1 & 9.182254704 & 0.178575988 & 0.101612624 \\
10 & 0.4 & 9.071426914 & 0.178564461 & 0.102854049 \\
10 & 0.8 & 8.726039146 & 0.178527017 & 0.106925144 \\
100 & 0.1 & 38.546073475 & 0.180136006 & 0.024205656 \\
100 & 0.4 & 38.050155049 & 0.180132872 & 0.024521135 \\
100 & 0.8 & 36.506088964 & 0.180122662 & 0.025558284 \\
\hline
\end{tabular}

data for optimal asset allocation and consumption strategies.

\section{Conclusion}

In this paper, we study the portfolio selection problem in a general setting under CRRA utility functions: stochastic volatility, incomplete markets, finite investment horizons, and consumption choice. To the best of our knowledge, no explicit solution or numerical result is available in the literature for this setting. We present an accurate and efficient numerical method for optimal asset allocation and optimal consumption strategies. The optimal strategies are depended on a solution to a nonlinear and inhomogeneous partial differential equation which is derived from the portfolio selection problem. A three-level Crank-Nicolson finite difference scheme, which has second-order accuracy, is used to determine numerical solutions. In addition, we have used a technique to deal with the nonlinear term, which is one of our main contributions. We believe that the technique to deal with the nonlinear term could be applied to other similar numerical problems. The Crank-Nicolson algorithm also has been extended to third-order accuracy by performing Richardson's extrapolation. Some experiments are conducted to verify the performance of the proposed algorithm. Based on this algorithm, we present the first set of accurate numerical solutions of optimal strategies. Since the portfolio selection problem under stochastic volatility is an important issue in modern finance, the proposed algorithm will be useful for further theoretical research and for applications in the financial industry.

\section{Appendix}

A. Derivations for $f_{\tau}(0, v), f_{\tau \tau}(0, v)$, and $f_{\tau \tau \tau}(0, v)$

From (19), we obtain

$$
\begin{aligned}
f_{\tau}= & a_{1} v f_{v v}+\left(a_{2} v+a_{3}\right) f_{v}+a_{4} v \frac{f_{v}^{2}}{f}+\left(a_{5} v+a_{6}\right) f \\
& +\alpha^{(1 / \gamma)} a_{7} .
\end{aligned}
$$

By taking the derivative of (A.1) with respect to $\tau$, we obtain

$$
\begin{aligned}
f_{\tau \tau}= & a_{1} v f_{v v \tau}+\left(a_{2} v+a_{3}\right) f_{v \tau}+a_{4} v \frac{2 f_{v} f_{v \tau}}{f}-a_{4} v \frac{f_{v}^{2}}{f^{2}} f_{\tau} \\
& +\left(a_{5} v+a_{6}\right) f_{\tau} .
\end{aligned}
$$


By taking the derivative of (A.2) with respect to $\tau$, we obtain

$$
\begin{aligned}
f_{\tau \tau \tau}= & a_{1} v f_{v v \tau \tau}+\left(a_{2} v+a_{3}\right) f_{v \tau \tau}+a_{4} v \frac{2 f_{v \tau}^{2}}{f}+a_{4} v \frac{2 f_{v} f_{v \tau \tau}}{f} \\
& -4 a_{4} v \frac{f_{v} f_{v \tau}}{f^{2}} f_{\tau}+2 a_{4} v \frac{f_{v}^{2}}{f^{3}} f_{\tau}^{2}-a_{4} v \frac{f_{v}^{2}}{f^{2}} f_{\tau \tau} \\
& +\left(a_{5} v+a_{6}\right) f_{\tau \tau} .
\end{aligned}
$$

By setting $\tau=0$ in (A.1) and using the initial condition $f(0, v)=(1-\alpha)^{(1 / \gamma)}$, we obtain

$$
f_{\tau}(0, v)=\left(a_{5} v+a_{6}\right) f(0, v)+\alpha^{(1 / \gamma)} a_{7} .
$$

By setting $\tau=0$ in (A.2) and using the initial condition $f(0, v)=(1-\alpha)^{(1 / \gamma)}$ and (A.4), we obtain

$$
\begin{aligned}
f_{\tau \tau}(0, v)= & \left(a_{5}^{2} v^{2}+\left(a_{2} a_{5}+2 a_{5} a_{6}\right) v+a_{3} a_{5}+a_{6}^{2}\right) f(0, v) \\
& +\left(a_{5} v+a_{6}\right) \alpha^{(1 / \gamma)} a_{7} .
\end{aligned}
$$

By setting $\tau=0$ in (A.3) and using the initial condition $f(0, v)=(1-\alpha)^{(1 / \gamma)},($ A.4), and (A.5), we obtain

$$
f_{\tau \tau \tau}(0, v)=a_{5}^{3} v^{3}+a_{2} a_{3} a_{5}+3 a_{3} a_{6} a_{5}+a_{6}^{3} .
$$

\section{B. HARA Utility Setting}

In this appendix, the portfolio selection problem under HARA utility setting is considered. We would like to show that the proposed method could also be applied to this problem under HARA utility setting.

We assume that HARA utility functions $u_{1}(\cdot)$ and $u_{2}(\cdot)$ are used for consumption utility and terminal wealth utility, respectively:

$$
\begin{gathered}
u_{1}\left(c_{t}\right)=\frac{a_{c}(1-\gamma)}{\gamma}\left(\frac{c_{t}}{1-\gamma}+b_{c}\right)^{\gamma}, \\
u_{2}\left(w_{T}\right)=\frac{a_{w}(1-\gamma)}{\gamma}\left(\frac{w_{T}}{1-\gamma}+b_{w}\right)^{\gamma} .
\end{gathered}
$$

The market setting and investors' objective are the same as before which are given by (2)-(6). Following the procedure in this paper, the value function satisfies the following equation:

$$
\begin{aligned}
0= & \frac{(1-\gamma)^{2}}{\gamma}\left(a_{c} \alpha e^{-\beta t}\right)^{(1 / 1-\gamma)} V_{w}^{(\gamma / \gamma-1)}+\left((1-\gamma) b_{c}+r w\right) V_{w} \\
& +V_{t}+\kappa(\theta-v) V_{v}+\frac{1}{2} \xi^{2} v V_{v v}-\frac{1}{2} v \frac{\left(A V_{w}+\rho \xi V_{w v}\right)^{2}}{V_{w w}}
\end{aligned}
$$

and optimal strategies are given by

$$
\begin{aligned}
\varphi^{*} & =-\frac{A V_{w}+\rho \xi V_{w v}}{w V_{w w}}, \\
c^{*} & =(1-\gamma)\left[\left(\frac{V_{w}}{a_{c} \alpha e^{-\beta t}}\right)^{(1 / \gamma-1)}-b_{c}\right] .
\end{aligned}
$$

Based on the terminal condition and the scaling property of this problem, a reasonable trial solution form for $V$ is assumed:

$$
V(\tau, w, v)=e^{-\beta(T-\tau)} \frac{a_{w}(1-\gamma)}{\gamma}\left(\frac{w}{1-\gamma}+g(\tau)\right)^{\gamma} f(\tau, v)^{1-\gamma},
$$

where $g(\tau)$ and $f(\tau, v)$ need to be determined and $\tau=T-t$. The initial conditions of $g$ and $f$ are

$$
\begin{aligned}
g(0) & =b_{w}, \\
f(0, v) & =(1-\alpha)^{(1 / 1-\gamma)} .
\end{aligned}
$$

To determine $f$ and $g$, we substitute expression (B.4) into (B.2) and obtain

$$
\begin{aligned}
& -f_{\tau}+\frac{1}{2} \xi^{2} v f_{v v}+\left(\kappa(\theta-v)+\frac{\gamma}{1-\gamma} A \rho \xi v\right) f_{v} \\
& -\frac{1}{2} \gamma\left(1-\rho^{2}\right) \xi^{2} v \frac{f_{v}^{2}}{f} \\
& +\left(\frac{A^{2} \gamma}{2(1-\gamma)^{2}} v+\frac{\gamma r}{1-\gamma}-\frac{\beta}{1-\gamma}\right) f+\left(\frac{\alpha a_{c}}{a_{w}}\right)^{(1 / 1-\gamma)} \\
& -\frac{\gamma}{1-\gamma}\left(\frac{g^{\prime}(\tau)+r g(\tau)-b_{c}}{(w / 1-\gamma)+g}\right) f=0 .
\end{aligned}
$$

By setting

$$
g^{\prime}(\tau)+r g(\tau)-b_{c}=0,
$$

equation (B.6) becomes an equation involving $f$ only, namely,

$$
\begin{aligned}
& -f_{\tau}+\frac{1}{2} \xi^{2} v f_{v v}+\left(\kappa(\theta-v)+\frac{\gamma}{1-\gamma} A \rho \xi v\right) f_{v} \\
& -\frac{1}{2} \gamma\left(1-\rho^{2}\right) \xi^{2} v \frac{f_{v}^{2}}{f} \\
& +\left(\frac{A^{2} \gamma}{2(1-\gamma)^{2}} v+\frac{\gamma r}{1-\gamma}-\frac{\beta}{1-\gamma}\right) f+\left(\frac{\alpha a_{c}}{a_{w}}\right)^{(1 / 1-\gamma)}=0 .
\end{aligned}
$$

Thus, we have achieved dimension reduction by removing $w$ dependence in (B.2). The solution of $g$ is obtained from (B.7) and (B.5):

$$
g(\tau)=b_{w} e^{-r \tau}+\frac{b_{c}}{r}\left(1-e^{-r \tau}\right) .
$$


Equation (B.8) has the same form as (19), which could be solved numerically by applying the proposed algorithm the same as that in Section 3. Then, the optimal strategies could be obtained:

$$
\begin{aligned}
\varphi^{*} & =\left(1+\frac{1-\gamma}{w} g(\tau)\right)\left(\frac{A}{1-\gamma}+\rho \xi \frac{f_{v}}{f}\right), \\
\frac{c^{*}}{w} & =\left(\frac{\alpha a_{c}}{a_{w}}\right)^{(1 / 1-\gamma)} f^{-1}+\frac{1-\gamma}{w}\left(\left(\frac{\alpha a_{c}}{a_{w}}\right)^{(1 / 1-\gamma)} g(\tau) f^{-1}-b_{c}\right) .
\end{aligned}
$$

\section{Data Availability}

The data used to support the findings of this study are available from the corresponding author upon request.

\section{Conflicts of Interest}

The authors declare that they have no conflicts of interest.

\section{Acknowledgments}

The work of L. Ge was supported by the Fundamental Research Funds for the Central Universities (220110004005040120 and 220110001002020043). The work of Q. Zhang was supported by the Research Grants Council of the Hong Kong Special Administrative Region, China (project CityU 11335816).

\section{References}

[1] A. Buraschi, P. Porchia, and F. Trojani, "Correlation risk and optimal portfolio choice," The Journal of Finance, vol. 65, no. 1, pp. 393-420, 2010.

[2] Š. Raudys, A. Raudys, and Ž. Pabarškaite,, "Sustainable economy inspired large-scale feed-forward portfolio construction," Technological and Economic Development of Economy, vol. 20, no. 1, pp. 79-96, 2014.

[3] J. Kallsen and J. Muhle-Karbe, "Utility maximization in affine stochastic volatility models," International Journal of Theoretical and Applied Finance, vol. 13, no. 3, pp. 459-477, 2010.

[4] P. Christoffersen, S. Heston, and K. Jacobs, "The shape and term structure of the index option smirk: why multifactor stochastic volatility models work so well," Management Science, vol. 55, no. 12, pp. 1914-1932, 2009.

[5] S. L. Heston, "A closed-form solution for options with stochastic volatility with applications to bond and currency options," Review of Financial Studies, vol. 6, no. 2, pp. 327343, 1993.

[6] S. Li, Y. Zhou, Y. Wu, and X. Ge, "Equilibrium approach of asset and option pricing under Lévy process and stochastic volatility," Australian Journal of Management, vol. 42, no. 2, pp. 276-295, 2017.

[7] S. Liu, Y. Zhou, Y. Wu, and X. Ge, "Option pricing under the jump diffusion and multifactor stochastic processes," Journal of Function Spaces, vol. 2019, Article ID 9754679, 12 pages, 2019.

[8] T. S. Kim and E. Omberg, "Dynamic nonmyopic portfolio behavior," Review of Financial Studies, vol. 9, no. 1, pp. 141-161, 1996.
[9] J. A. Wachter, "Portfolio and consumption decisions under mean-reverting returns: an exact solution for complete markets," The Journal of Financial and Quantitative Analysis, vol. 37, no. 1, pp. 63-91, 2002.

[10] J. Liu, "Portfolio selection in stochastic environments," Review of Financial Studies, vol. 20, no. 1, pp. 1-39, 2007.

[11] Y. Xia, "Learning about predictability: the effects of parameter uncertainty on dynamic asset allocation," The Journal of Finance, vol. 56, no. 1, pp. 205-246, 2001.

[12] M. Schroder and C. Skiadas, "Optimal consumption and portfolio selection with stochastic differential utility," Journal of Economic Theory, vol. 89, no. 1, pp. 68-126, 1999.

[13] G. Chacko and L. M. Viceira, "Dynamic consumption and portfolio choice with stochastic volatility in incomplete markets," Review of Financial Studies, vol. 18, no. 4, pp. 1369-1402, 2005.

[14] R. I. Palwasha, N. Ahmad, R. R. Ahmed, J. Vveinhardt, and D. Streimikiene, "Speed of mean reversion: an empirical analysis of kse, lse and ise indices," Technological and Economic Development of Economy, vol. 24, no. 4, pp. 1435-1452, 2018.

[15] R. C. Merton, "On estimating the expected return on the market," Journal of Financial Economics, vol. 8, no. 4, pp. 323-361, 1980.

[16] K. R. French, G. W. Schwert, and R. F. Stambaugh, "Expected stock returns and volatility," Journal of Financial Economics, vol. 19, no. 1, pp. 3-29, 1987.

[17] H. Kraft, "Optimal portfolios and Heston's stochastic volatility model: an explicit solution for power utility," Quantitative Finance, vol. 5, no. 3, pp. 303-313, 2005.

[18] D. He, "On the Lo-norm convergence of a three-level linearly implicit finite difference method for the extended FisherKolmogorov equation in both $1 \mathrm{D}$ and 2D," Computers \& Mathematics with Applications, vol. 71, no. 12, pp. 2594-2607, 2016.

[19] B. Wongsaijai and K. Poochinapan, "A three-level average implicit finite difference scheme to solve equation obtained by coupling the Rosenau-KdV equation and the Rosenau-RLW equation," Applied Mathematics and Computation, vol. 245, pp. 289-304, 2014.

[20] D. He, "Exact solitary solution and a three-level linearly implicit conservative finite difference method for the generalized Rosenau-Kawahara-RLW equation with generalized Novikov type perturbation," Nonlinear Dynamics, vol. 85, no. 1, pp. 479-498, 2016.

[21] H.-Y. Cao, Z.-Z. Sun, and G.-H. Gao, "A three-level linearized finite difference scheme for the camassa-holm equation," Numerical Methods for Partial Differential Equations, vol. 30, no. 2, pp. 451-471, 2014.

[22] S. Frederick, G. Loewenstein, and T. O'donoghue, "Time discounting and time preference: a critical review," Journal of Economic Literature, vol. 40, no. 2, pp. 351-401, 2002.

[23] C. Munk, Financial Asset Pricing Theory, Oxford University Press, Oxford, UK, 2013. 